\title{
Patterns and Effects of Admission Hyperglycemia and Inflammatory Response in Trauma Patients: A Prospective Clinical Study
}

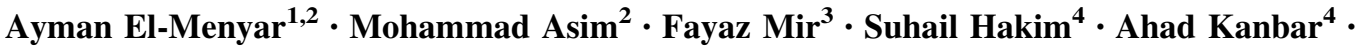 \\ Tariq Siddiqui $^{4} \cdot$ Basil Younis $^{4} \cdot$ Khalid Ahmed $^{4} \cdot$ Ismail Mahmood $^{4} \cdot$ Sajid Atique $^{4} \cdot \mathrm{Hisham} \mathrm{Al} \mathrm{Jogol}^{4}$. \\ Ibrahim Taha ${ }^{4} \cdot$ Fuad Mustafa $^{4} \cdot$ Mohammad Alabdallat $^{4} \cdot$ Husham Abdelrahman $^{4} \cdot$ Ruben Peralta $^{4,5}$. \\ Hassan Al-Thani ${ }^{4}$
}

Accepted: 13 May 2021/Published online: 11 June 2021

(C) The Author(s) 2021, corrected publication 2021

\begin{abstract}
Background The constellation of the initial hyperglycemia, proinflammatory cytokines and severity of injury among trauma patients is understudied. We aimed to evaluate the patterns and effects of on-admission hyperglycemia and inflammatory response in a level 1 trauma center. We hypothesized that higher initial readings of blood glucose and cytokines are associated with severe injuries and worse in-hospital outcomes in trauma patients.

Methods A prospective, observational study was conducted for adult trauma patients who were admitted and tested for on-admission blood glucose, hemoglobin A1c, interleukin (IL)-6, IL-18 and hs-CRP. Patients were categorized into four groups [non-diabetic normoglycemic, diabetic normoglycemic, diabetic hyperglycemic (DH) and stressinduced hyperglycemic $(\mathrm{SIH})]$. The inflammatory markers were measured on three time points (admission, $24 \mathrm{~h}$ and $48 \mathrm{~h}$ ). Generalized estimating equations (GEE) were used to account for the correlation for the inflammatory markers. Pearson's correlation test and logistic regression analysis were also performed.

Results During the study period, 250 adult trauma patients were enrolled. Almost $13 \%$ of patients presented with hyperglycemia (50\% had SIH and 50\% had DH). Patients with SIH were younger, had significantly higher Injury Severity Score (ISS), higher IL-6 readings, prolonged hospital length of stay and higher mortality. The SIH group had lower Revised Trauma Score $(p=0.005)$, lower Trauma Injury Severity Score $(p=0.01)$ and lower GCS $(p=0.001)$. Patients with hyperglycemia had higher in-hospital mortality than the normoglycemia group (12.5\% vs $3.7 \% ; p=0.02$ ). A significant correlation was identified between the initial blood glucose level and serum lactate, IL-6, ISS and hospital length of stay. Overall rate of change in slope 88.54 (95\% CI:-143.39-33.68) points was found more in hyperglycemia than normoglycemia group $(p=0.002)$ for IL-6 values, whereas there was no statistical significant change in slopes of age, gender and their interaction. The initial IL-6 levels correlated with ISS ( $r=0.40$, $p=0.001$ ). On-admission hyperglycemia had an adjusted odds ratio 2.42 (95\% CI: 1.076-5.447, $p=0.03$ ) for severe injury (ISS > 12) after adjusting for age, shock index and blood transfusion.

Conclusions In trauma patients, on-admission hyperglycemia correlates well with the initial serum IL-6 level and is associated with more severe injuries. Therefore, it could be a simple marker of injury severity and useful tool for patient triage and risk assessment.

Trial registration This study was registered at the ClinicalTrials.gov (Identifier: NCT02999386), retrospectively Registered on December 21, 2016. https://clinicaltrials.gov/ct2/show/NCT02999386.
\end{abstract}

Supplementary Information The online version contains supplementary material available at https://doi.org/10.1007/s00268021-06190-5.

Ayman El-Menyar aymanco65@yahoo.com
1 Clinical Medicine, Weill Cornell Medical College, Doha, Qatar

2 Trauma \& Vascular Surgery, Clinical Research, Hamad General Hospital, Doha, Qatar 


$\begin{array}{ll}\text { Abbreviations } \\ \text { ISS } & \text { Injury severity score } \\ \text { hs-CRP } & \text { High-sensitive reactive protein } \\ \text { IL } & \text { Interleukin } \\ \text { HbAlc } & \text { Glycosylated hemoglobin } \\ \text { SIH } & \text { Stress-induced hyperglycemia } \\ \text { DH } & \text { Diabetic hyperglycemia }\end{array}$

\section{Introduction}

Hyperglycemia following trauma is a hypermetabolic response to stress which can be associated with a significant morbidity and mortality [1]. Some investigators have suggested that admission glucose level could be used as a potential predictor of hospital outcomes as it reflects the physiological stress reaction to injury, bleeding and shock [2-4]. However, there is still a need to have a consensus on the definition and measurement of stress-induced hyperglycemia (SIH) in trauma patients [2].

The trauma-related metabolic surge and associated $\mathrm{SIH}$ were found to correlate with serum cortisol and catecholamine levels [5]. It has been suggested that insulin production is suppressed in trauma patients due to systemic stress response secondary to elevated serum glucagon, catecholamine and cytokines [6-8].

Assessment of glycosylated hemoglobin (HbA1c) level is considered as a useful tool to distinguish occult (not known before) diabetes mellitus (DM) from SIH [9]. Kopelman et al. [10] reported that $18 \%$ of trauma patients initially presented with hyperglycemia, of which $22 \%$ had occult DM which represented $4 \%$ of the total screened trauma patients. The possible mechanism of the adverse effects of hyperglycemia may differ in patients with SIH as compared to diabetic hyperglycemia (DH). SIH is an acute process, initiated by the release of stress hormones and cytokines, while DH is a chronic condition associated with subsequent microvascular changes [11].

Moreover, there is a relationship between hyperglycemia and altered cellular metabolism in critically ill patients that results in insulin resistance and release of systemic inflammatory mediators [6]. Earlier studies have suggested that proinflammatory cytokines such as interleukin (IL)-6 and IL-18 are involved in glucose

3 Qatar Metabolic Institute, Hamad Medical Corporation, Doha, Qatar

4 Department of Surgery, Trauma Surgery, Hamad General Hospital, Doha, Qatar

5 Department of Surgery, Universidad Nacional Pedro Henriquez Urena, Santo Domingo, Dominican Republic metabolism and insulin action; therefore, hypercytokinemia may have a potential role in increased glucose levels [6, 12-14]. As most observations on the association of cytokines with hyperglycemia are based on experimental studies, there is a need to explore such relationship with respect to the clinical presentation and outcome in trauma patients. Herein, this prospective study aims to evaluate the patterns and effects of on-admission hyperglycemia, proinflammatory cytokines and severity of injury in trauma patients. We hypothesized that higher initial readings of blood glucose and cytokines are associated with severe injuries and worse in-hospital outcomes in trauma patients.

\section{Materials and methods}

A prospective observational study was conducted for trauma patients who were admitted to the level 1 trauma center at Hamad General Hospital (HGH) between October 2016 and July 2019. Inclusion criteria were adult ( $\geq 18$ years) trauma patients (all genders) presented to the emergency department and were investigated for random blood glucose level and HbA1C within $5 \mathrm{~h}$ of hospital admission. Exclusion criteria included patients declined to participate or in whom random glucose level and HbA1C were not measured on time, vulnerable populations (children, pregnant women) and alcohol consumers. All trauma patients underwent thorough clinical assessment and resuscitation according to the Advanced Trauma Life Support (ATLS) guidelines. Potential subjects were enrolled after obtaining written informed consent either by subject or his/her next-of-kin or deferred consent for blood investigations and use of data with secured confidentiality of personal information.

\section{Study variables}

Data included patients' demographics, (age, gender, nationality), mechanism of injury, initial vitals (heart rate, respiratory rate, systolic blood pressure, diastolic blood pressure and shock index), routine laboratory findings such as hemoglobin, base deficit, serum lactate and blood glucose levels were recorded at the baseline, after $24 \mathrm{~h}$ and $48 \mathrm{~h}$. Other investigations included white blood cell count (WBC), platelet count, high-sensitive troponin T (hs-TnT), HbA1C and blood ethanol levels. We have collected information about history of DM, anti-diabetic medications, associated injuries, Injury Severity Score (ISS), Glasgow Coma Score (GCS), Revised Trauma Score (RTS), Trauma Injury Severity Score (TRISS), surgical intervention, blood transfusion, hospital length of stay, intensive care unit stay, in-hospital complications (pneumonia, acute respiratory distress syndrome, renal failure 
and sepsis) and hospital mortality. Shock index (SI) was defined as initial heart rate divided by the initial systolic blood pressure.

The main exposure was hyperglycemia, defined as random serum glucose $200 \mathrm{mg} / \mathrm{dL}(11.1 \mathrm{mmol} / \mathrm{l})$ or more. This cutoff level of glucose was previously used to define hyperglycemia by earlier studies in trauma patients [15-17]. DM was determined by patient history and/or admission $\mathrm{HbA} 1 \mathrm{c} \geq 6.5 \%$ ( $\geq 48 \mathrm{mmol} / \mathrm{mol})$. This level of $\mathrm{HbAlc}$ is based on current recommendations for the diagnosis of DM from the American Diabetes Association [18]. SIH was defined as hyperglycemia on admission in patients with normal HbAlc in the index admission [19].

\section{Serum levels of CRP, IL-6 and IL-18}

Enzyme-linked immunosorbent assay (ELISA) was performed using commercially available kits for cytokine detection (R\&D) Systems. The preparation of all reagents, the working standards and protocol were followed according to the manufacturer's instructions. The absorbance was read using ELISA reader (TECAN) at $450 \mathrm{~nm}$ and $570 \mathrm{~nm}$ using dual filters. The minimum detectable dose was $0.005 \mathrm{ng} / \mathrm{ml}$ for hs-CRP, $0.7 \mathrm{pg} / \mathrm{ml}$ for IL-6 and $1.25 \mathrm{pg} / \mathrm{ml}$ for IL-18. All the samples were thawed only once and assayed in duplicate.

\section{Statistical analysis}

Sample size was calculated considering the prevalence of $\mathrm{SIH}$ in trauma patients that ranges from 10 to $17 \%$ for all trauma admissions $[15,20]$ with a precision of estimate (margin of error) of 5\% and a 95\% level of confidence. Using the single proportion equation for dichotomous variables in the nMaster 2.0 sample size software package, the required sample size was 250 consecutive trauma patients.

Data were reported as proportion, mean $( \pm$ standard deviation), confidence intervals, median and interquartile range (IQR), whenever applicable. Assessment of normality of continuous data was performed using ShapiroWilk test. The mean values of IL-6, IL-18, hs-CRP and blood glucose at baseline after $24 \mathrm{~h}$ and $48 \mathrm{~h}$ were expressed as mean and $95 \%$ confidence interval (95\% CI). The levels of blood glucose, serum lactate, base excess, IL6, IL-18, hs-CRP, complications and outcome were compared based on the ISS. Patients were first compared as two groups based on the initial glucose levels (normoglycemic vs hyperglycemic). Then, all patients were divided into four subgroups based on the initial glucose and HbA1c values: (1) non-diabetic normoglycemic, (2) SIH, (3) diabetic normoglycemic and (4) DH (Fig. 1). The study groups were compared using $\chi^{2}$ test for categorical variables and the one-way analysis of variance (ANOVA) or Student's $t$ test for comparison of continuous variables whenever applicable. Yates' corrected chi-square was used for categorical variables if the expected cell frequencies were below 5. A significant difference was considered when the two-tailed $p$ value was less than 0.05 . Freedmen ANOVAs, a nonparametric repeated measure analysis of variance, were performed separately for normoglycemia and hyperglycemia group to see the trend within the group

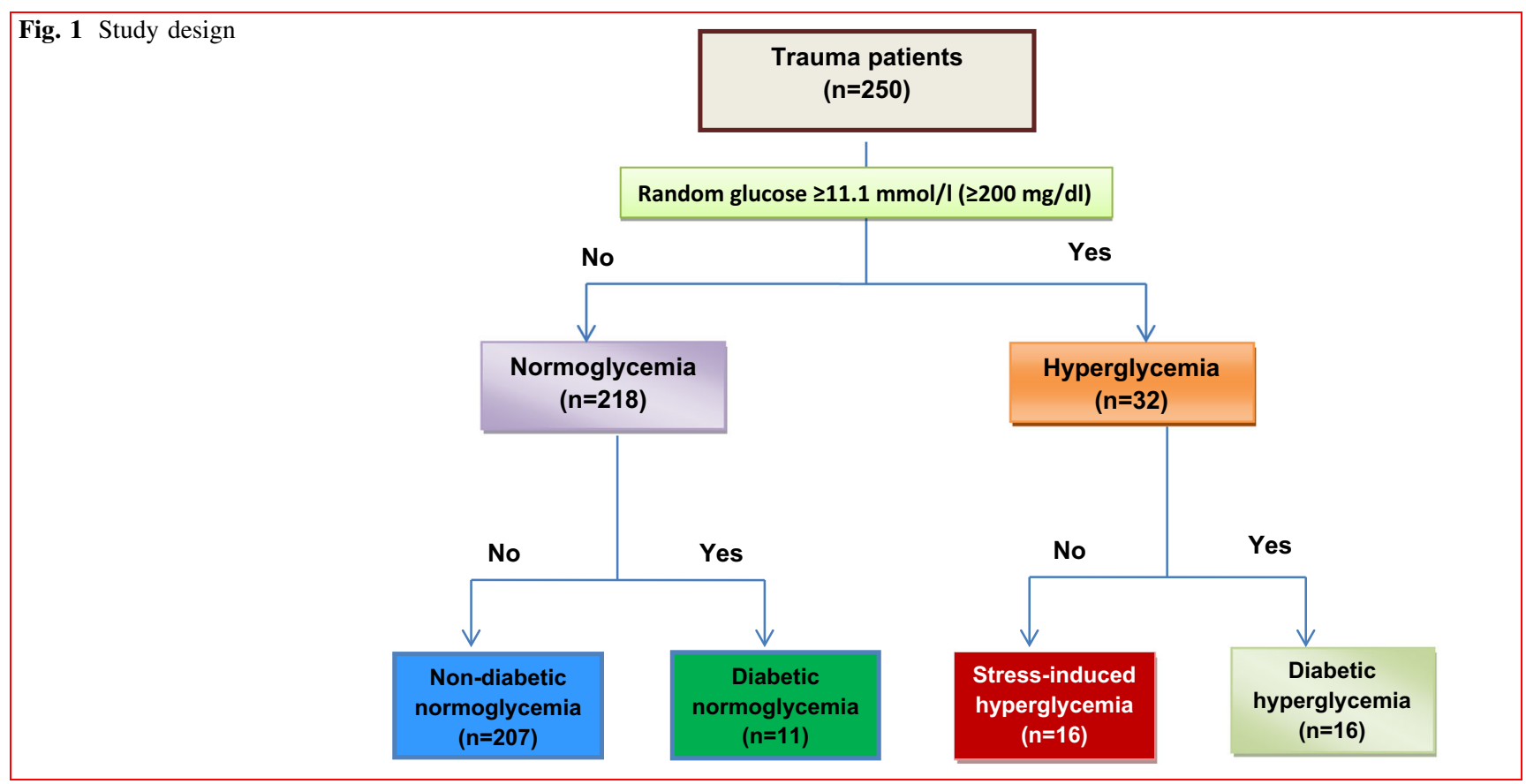


Table 1 Analysis of demographics, clinical presentation and outcome of trauma patients according to blood glucose level (October 2016-July 2019)

\begin{tabular}{|c|c|c|c|c|}
\hline & Overall $(n=250)$ & Normoglycemia $n=218(87 \%)$ & Hyperglycemia* $n=32(13 \%)$ & $\mathrm{p}$ value \\
\hline Age; years $(n=250)^{\dagger}$ & $35.1 \pm 10.1$ & $34.9 \pm 10.1$ & $36.7 \pm 9.6$ & 0.33 \\
\hline \multicolumn{5}{|l|}{ Gender $(n=250)$} \\
\hline Males & $245(98.0 \%)$ & $214(98.2 \%)$ & $31(96.9 \%)$ & 0.62 \\
\hline Females & $5(2.0 \%)$ & $4(1.8 \%)$ & $1(3.1 \%)$ & 0.62 \\
\hline Body mass index $(n=248)^{\dagger}$ & $26.3 \pm 11.1$ & $25.8 \pm 8.7$ & $29.8 \pm 20.9$ & 0.29 \\
\hline hs-TnT positive $(n=235)$ & $49(20.9 \%)$ & $33(16.2 \%)$ & $16(51.6 \%)$ & 0.001 \\
\hline Fasting glucose after $24 \mathrm{~h}(n=247)^{* *}$ & $7.25(6.92-7.58)$ & $6.9(6.6-7.2)$ & $9.2(7.9-10.4)$ & 0.001 \\
\hline Fasting glucose after $48 \mathrm{~h}(n=237)^{* *}$ & $6.38(6.13-6.63)$ & $6.2(5.9-6.4)$ & $7.5(6.4-8.6)$ & 0.006 \\
\hline Hemoglobin A1c $\%(n=250)^{* *}$ & $5.61(5.47-5.76)$ & $5.4(5.3-5.5)$ & $6.9(6.1-7.8)$ & 0.003 \\
\hline Injury Severity Score $(n=250)^{* *}$ & $14.7(13.4-15.9)$ & $13.5(12.314 .7)$ & $22.6(17.5-27.7)$ & 0.001 \\
\hline Revised Trauma Score $(n=205)^{\dagger}$ & $7.45 \pm 1.13$ & $7.6 \pm 0.9$ & $6.6 \pm 1.7$ & 0.005 \\
\hline $\operatorname{TRISS}(n=198)^{\dagger}$ & $0.9566 \pm 0.1262$ & $0.9713 \pm 0.1011$ & $0.8681 \pm 0.2065$ & 0.01 \\
\hline Hospital length of stay $(n=250)^{£}$ & $8(2-146)$ & $8(2-68)$ & $12.5(3-146)^{* *}$ & 0.001 \\
\hline ICU length of stay $(n=103)^{£}$ & $4(1-40)$ & $4(1-40)$ & $6(2-23)$ & 0.04 \\
\hline Blood transfusion $(n=250)$ & $70(28.0 \%)$ & $56(25.7 \%)$ & $14(43.8 \%)$ & 0.03 \\
\hline Blood units transfused $(n=70)^{£}$ & $2.5(1-68)$ & $2(1-22)$ & $6(1-68)$ & 0.03 \\
\hline Hospital complications $(n=250)$ & $8(3.2 \%)$ & $6(2.8 \%)$ & $2(6.3 \%)$ & 0.29 \\
\hline Hospital mortality $(n=250)$ & $12(4.8 \%)$ & $8(3.7 \%)$ & $4(12.5 \%)$ & 0.02 \\
\hline
\end{tabular}

${ }^{*}$ Random blood glucose $\geq 11.1 \mathrm{mmol} / 1$ at baseline, TRISS: trauma injury severity score, Data presented as ${ }^{\dagger}$ mean $\pm \mathrm{SD}$, or $* *$ mean and $95 \%$ $\mathrm{CI},{ }^{£}$ median and range. Statistical tests used: Student $\mathrm{t}$ tests and Mann-Whitney $\mathrm{U}$ tests were performed for interval variables between the two groups (Normoglycemia and Hyperglycemia) wherever applicable. Chi-square tests with Yate's correction factors were used for categorical variables to see associations between the two groups

for IL-6, IL-18, hs-CRP and blood glucose at baseline, after $24 \mathrm{~h}$ and $48 \mathrm{~h}$, whereas Mann-Whitney $\mathrm{U}$ tests were used to see significant difference between the groups at each point of time and Wilcoxon signed-rank tests to see significant differences within each group from baseline for univariate exploratory analysis. Mixed model regression analyses were performed in the form of generalized estimating equations (GEE) to deal with longitudinal data and to adjust covariates. Cofactors as age and gender were adjusted along with groups (normoglycemia and hyperglycemia) and time variable for interleukin and hs-CRP values. Separate GEE analysis was performed using compound symmetry correlation structure.

Multivariable logistic regression analysis was performed to determine the odds ratio of on-admission blood glucose for the injury severity (ISS $>12$ ) in one model after adjusting for age, shock index and blood transfusion and for hospital mortality in another model after adjusting for age, GCS, ISS, shock index and blood transfusion. Correlation of initial blood glucose level with respect to initial WBC count, lactate and base excess, ISS, RTS, TRISS, hospital length of stay and inflammatory markers was performed using Pearson's correlation. Data analysis was carried out using the SPSS version 18 (SPSS Inc., Chicago,
Illinois). Figures were plotted using GraphPad Prism software V.9.0 (La Jolla, CA).

\section{Results}

\section{Patient and injury characteristics}

During the study period, 250 trauma patients were enrolled. Figure 1 shows the study design. The vast majority of patients were males (98.0\%) with mean age of $35.1 \pm 10.1$ years. The mean body mass index (BMI) was $26.3 \pm 11.1 \mathrm{~kg} / \mathrm{m}^{2}$, systolic blood pressure was $124.9 \pm 20.7 \mathrm{mmHg}$ and diastolic blood pressure was $78.2 \pm 13.9 \mathrm{mmHg}$. The frequently injured body region was lower extremity $(45.2 \%)$ followed by chest (40.4\%), head $(30.0 \%)$ and upper extremities $(29.6 \%)$. The average glucose level at baseline was $8.38 \mathrm{mmol} / \mathrm{l}(95 \% \mathrm{CI}$; 8.01-8.74) and HbA1c was 5.61 (5.47-5.76). The mean ISS was 14.7 (95\% CI 13.4-15.9), mean RTS score was $7.45 \pm 1.13$ and mean TRISS was $0.9566 \pm 0.12$. Blood transfusion was required in $28 \%$ cases and $3.2 \%$ developed in-hospital complications. The overall hospital mortality was $4.8 \%$ (12 patients). 
Table 2 Trend analysis for Interleukin-6, Interleukin-18 and high-sensitive C-reactive protein (hs-CRP)

\begin{tabular}{|c|c|c|c|c|}
\hline Variables & Overall & Normoglycemia & Hyperglycemia & $p$ value between the twogroups \\
\hline \multicolumn{5}{|l|}{ Interleukin-6 (pg/ml) } \\
\hline Baseline (on-admission) $(n=250)$ & $137(121-153)$ & $122(107-137)$ & $240(172-308)$ & 0.001 \\
\hline Second day (after 24 h) $(n=250)$ & $120(101-138)$ & $112(93-131)^{\mathrm{a}}$ & $172(109-236)^{\mathrm{g}}$ & 0.02 \\
\hline Third day (after 48 h) $(n=236)$ & $108(90-125)$ & $94(78-111)^{\mathrm{b}}$ & $193(127-259)^{\mathrm{k}}$ & 0.008 \\
\hline$p$ value within the group & - & 0.001 & 0.02 & \\
\hline \multicolumn{5}{|l|}{ Interleukin-18 (pg/ml) } \\
\hline Baseline (on-admission) $(n=250)$ & $305(238-371)$ & $299(226-372)$ & $369(128-610)$ & 0.11 \\
\hline Second day (after 24 h) $(n=250)$ & 218(199-236) & $218(197-238)^{\mathrm{c}}$ & $217(186-248)^{1}$ & 0.28 \\
\hline Third day (after 48 h) $(n=236)$ & 215(198-233) & $211(192-231)^{\mathrm{d}}$ & $242(194-291)^{\mathrm{m}}$ & 0.05 \\
\hline$p$ value within the group & - & 0.64 & 0.20 & \\
\hline \multicolumn{5}{|l|}{$H s-C R P(\mathrm{ng} / \mathrm{ml})$} \\
\hline Baseline (on-admission) $(n=250)$ & $3.86(3.45-4.26)$ & $3.9(3.4-4.3)$ & $3.9(2.7-5.1)$ & 0.94 \\
\hline Second day $($ after 24 h) $(n=250)$ & $9.50(9.03-9.97)$ & $9.4(8.9-9.9)^{\mathrm{e}}$ & $10.1(8.9-11.3)^{\mathrm{n}}$ & 0.46 \\
\hline Third day (after 48 h) $(n=236)$ & $9.64(9.14-10.13)$ & $9.5(9.0-10.1)^{\mathrm{f}}$ & $10.3(9.0-11.5)^{\mathrm{o}}$ & 0.43 \\
\hline$p$ value within the group & - & 0.001 & 0.001 & \\
\hline
\end{tabular}

${ }^{\mathrm{a}}$ Baseline vs Second day; $p=0.008,{ }^{\mathrm{b}}$ Baseline vs third day; $p=0.001,{ }^{\mathrm{c}}$ Baseline vs Second day; $p=0.17 ;{ }^{\mathrm{d}}$ Baseline vs third day; $p=0.18,{ }^{\mathrm{e}}$ Baseline vs Second day; $p=0.001,{ }^{\mathrm{f}}$ Baseline vs third day; $p=0.001,{ }^{\mathrm{g}}$ Baseline vs Second day; $p=0.009,{ }^{\mathrm{k}}$ Baseline vs third day; $p=0.07,{ }^{1}$ Baseline vs Second day; $p=0.16,{ }^{\mathrm{m}}$ Baseline vs third day; $p=0.51,{ }^{\mathrm{n}}$ Second day; $p=0.001$, ${ }^{\mathrm{o}}$ Baseline vs third day; $p=0.001$. Baseline vs Data are given as mean and 95\% confidence intervals within the group, whereas Mann-Whitney U tests were used to see significant difference between the groups at each point of time, and Wilcoxon signed-rank tests were used to see significance differences within each group from baseline for univariate exploratory within the group, whereas Mann-Whitney U tests were used to see significant difference between the groups at each point of time, and Wilcoxon signed-rank tests were used to see significance differences within each group from baseline for univariate exploratory analysis.

\section{Normoglycemia vs hyperglycemia}

In the trauma cohort $(n=250), 13 \%$ of the patients had hyperglycemia on their initial presentation, of which $50 \%$ had SIH and 50\% had diabetic hyperglycemia.

Table 1 compares the demographics, clinical presentation and outcome of trauma patients according to the initial blood glucose levels (hyperglycemia versus normoglycemia). The two groups were comparable for age, gender and BMI. Compared with the normoglycemia group, hyperglycemic patients had higher HbAlc and positive hs-TnT $(51.6 \%$ vs $16.2 \% ; p=0.001)$. In the hyperglycemia group, serum concentrations of IL-6 at baseline, at $24 \mathrm{~h}$ and $48 \mathrm{~h}$ post-trauma, were significantly higher than those of the normoglycemia group. Moreover, the hyperglycemia group had a significantly higher mean ISS 22.6 (95\% CI 17.5-27.7 vs 13.5 (95\% CI 12.3-14.7); $p=0.001)$, lower RTS $(6.6 \pm 1.7$ vs $7.6 \pm 0.9 ; p=0.005)$ and TRISS scores $(0.8681 \pm 0.21$ vs $0.9713 \pm 0.10$; $p=0.01)$ and required more blood transfusion $(43.8 \%$ vs $25.7 \% ; p=0.03)$ than the normoglycemia group.

The hyperglycemia group had prolonged ICU $(p=0.001)$ and hospital stay $(p=0.001)$. Patients with hyperglycemia had higher mortality than normoglycemia group with $8.8 \%$ difference in death rate $(12.5 \%$ vs $3.7 \%$; $p=0.02$ ).

Table 2 shows trend analysis of the laboratory parameters IL-6, IL-18 and hs-CRP, at three time points for each group. IL-6 values changes were significant between the normoglycemia and hyperglycemia groups and within the variable for each group. The IL-6 levels were significantly decreasing as time increasing for both the groups $(p<0.05)$, and the variable values were statistical more in the hyperglycemia than the normoglycemia group at each point of time $(p<0.05)$ (Table3 \& Suppl Fig. 1). There was no statistically significant change between and within IL-18 levels. Notably, for hs-CRP levels, there were increases from the baseline to day 2 and 3 in the normoglycemia and hyperglycemia group $(p<0.05)$, however, there was no statistically significant difference at any point of time between the two groups.

\section{Analysis of the four subgroups}

The four trauma patients' groups were DH $(n=16)$, SIH $(n=16)$, diabetic normoglycemia $(n=11)$ and non-diabetic normoglycemia $(n=207)$ as shown in Table 4 . Compared to the other three groups, patients with SIH were significantly younger (mean age 32 years), more obese, had 
Table 3 GEE analysis for Interleukin-6 and hs-C-reactive protein (hs-CRP)

\begin{tabular}{|c|c|c|c|c|c|c|c|}
\hline \multicolumn{8}{|c|}{ Parameter estimates } \\
\hline \multirow[t]{2}{*}{ Interleukin-6 } & \multirow[t]{2}{*}{$\beta$} & \multirow[t]{2}{*}{ Standard error } & \multicolumn{2}{|c|}{$95 \%$ Wald confidence interval } & \multicolumn{3}{|l|}{ Hypothesis test } \\
\hline & & & Lower & Upper & Wald chi-square & df & $p$ value \\
\hline \multicolumn{8}{|c|}{ 1. GEE analysis for interleukin- 6 between and within factors } \\
\hline (Intercept) & 116.182 & 205.6032 & -286.793 & 519.157 & 0.319 & 1 & 0.572 \\
\hline [group $=0.00]$ & -88.54 & 27.9884 & -143.395 & -33.683 & 10.007 & 1 & 0.002 \\
\hline [group $=1.00]$ & $0^{\mathrm{a}}$ & & & & & & \\
\hline Gender & 39.977 & 203.8760 & -359.613 & 439.566 & 0.038 & 1 & 0.845 \\
\hline Age & 3.657 & 7.1711 & -10.398 & 17.712 & 0.260 & 1 & 0.610 \\
\hline (Scale) & $18,002.161$ & & & & & & \\
\hline \multicolumn{8}{|c|}{ Parameter estimates } \\
\hline \multirow[t]{2}{*}{ Parameter } & B & Std. error & \multicolumn{2}{|c|}{$95 \%$ Wald confidence interval } & \multicolumn{3}{|l|}{ Hypothesis test } \\
\hline & & & Lower & Upper & Wald chi-square & df & $p$ value \\
\hline \multicolumn{8}{|c|}{ 2. GEE for hs-CRP between and within factors } \\
\hline (Intercept) & 12.977 & 4.3424 & 4.466 & 21.488 & 8.931 & 1 & 0.003 \\
\hline [group $=.00]$ & -0.477 & 0.4851 & -1.428 & 0.474 & 0.965 & 1 & 0.326 \\
\hline [group = 1.00] & $0^{\mathrm{a}}$ & & & & & & \\
\hline Age & -0.150 & 0.1039 & -0.354 & 0.053 & 2.088 & 1 & 0.148 \\
\hline Gender & -4.860 & 4.3566 & -13.399 & 3.679 & 1.244 & 1 & 0.265 \\
\hline (Scale) & 20.175 & & & & & & \\
\hline
\end{tabular}

Dependent Variable: Interleukin-6,

Model: (Intercept), group, Gender, Age, gender * age

a. Reference

Dependent Variable: Hs-C-reactive values

Model: (Intercept), group, Age, Gender, Age * Gender

a. Reference

Group 0 = normoglycemia, group 1 = hyperglycemia. Interleukin- 6 and hs-CRP values were showing significant trend at univariate analysis. GEE multivariate mixed method was used to see trend analysis after adjusting age and gender confounders

severe injuries [mean ISS 24.5 (18-31)]; higher IL-6 levels at the three time points, prolonged hospital length of stay and higher mortality $(p=0.005)$. However, the four groups were comparable in terms of serum IL-18 and C-reactive protein levels at the different time points. Patients with diabetic hyperglycemia had higher hs-TnT and needed more units of blood transfusion. Higher shock index $(>0.80)$ was observed in both types of hyperglycemia compared to normoglycemic groups.

Figure 2 demonstrates the trend of inflammatory markers and blood glucose levels in trauma patients. The serum levels of IL-6, IL-18 and blood glucose increased after injury then showed a slowly decreasing trend, but did not reach baseline after $48 \mathrm{~h}$.

Table 5 illustrates the bivariate correlation between blood glucose levels and other factors. Significant positive correlations were identified between initial blood glucose level and serum lactate $(r=0.467, p=0.001)$, ISS $(r=0.368, p=0.001)$, IL-6 $(r=0.373, p=0.001)$ and hospital length of stay $(\mathrm{r}=0.304, p=0.001)$, whereas blood glucose levels showed significant negative correlations with base excess $(r=-0.417, p=0.001)$, GCS score $(r=-0.306, p=0.001)$, TRISS $(r=-0.310, p=0.001)$ and RTS $(r=-0.354, p=0.001)$. IL-6 correlated well with ISS $(r=0.40, p=0.001)$.

Table 6 shows the laboratory parameters and outcomes based on ISS. Compared to mild and moderate injury, patients sustained severe injury (ISS $\geq 16$ ) were more likely to have higher level of blood glucose, serum lactate, base excess and IL-6 ( $p=0.001$ for all). However, the mean serum levels of IL-18 and hs-CRP, at three time points, did not significantly differ among the ISS groups.

Multivariable logistic regression analysis showed that on-admission hyperglycemia had an adjusted odds ratio 
Table 4 Comparative analysis of trauma patients based on the initial blood glucose and hemoglobin A1C

\begin{tabular}{|c|c|c|c|c|c|}
\hline & $\mathrm{NN}(n=207)$ & $\mathrm{ND}(n=11)$ & $\mathrm{SIH}(n=16)$ & $\mathrm{DH}(n=16)$ & $p$ value \\
\hline Age; years & $34(33-36)$ & $44(36-53)$ & $32(28-36)$ & $41(36-46)$ & 0.001 \\
\hline Body mass index & $26(24-27)$ & $26(23-28)$ & $34(19-50)$ & $25(23-27)$ & 0.030 \\
\hline Shock index & $0.72(0.66-0.78)$ & $0.71(0.55-0.87)$ & $0.87(0.72-1.01)$ & $0.88(0.69-1.07)$ & 0.290 \\
\hline Injury Severity Score & $13(12-14)$ & $19(10.5-28)$ & $24.5(18-31)$ & $21(12-29)$ & 0.001 \\
\hline Revised Trauma Score & $8(7-8)$ & $7(6-8)$ & $6(5-7)$ & $7(6-8)$ & 0.001 \\
\hline GCS on arrival & $14(13-14)$ & $12(9-15)$ & $10(7-13)$ & $12(9-14)$ & 0.001 \\
\hline $\mathrm{HbA} 1 \mathrm{c} \%$ & $5.3(5.2-5.4)$ & $7.3(6.02-8.65)$ & $5.2(4.95-5.37)$ & $8.7(7.52-9.81)$ & 0.001 \\
\hline High-sensitive TroponinT & $17(9-26)$ & $12(9-15)$ & $49(15-81)$ & $114(-56-285)$ & 0.003 \\
\hline \multicolumn{6}{|l|}{ Interleukin-6 (pg/ml) } \\
\hline On-admission & $122(106-107)$ & $130(55-204)$ & $258(144-372)$ & $222(135-308)$ & 0.001 \\
\hline After $24 \mathrm{~h}$ & 112(92-131) & $123(51-195)$ & $220(115-325)$ & $125(49-201)$ & 0.046 \\
\hline After $48 \mathrm{~h}$ & $93(76-110)$ & $113(20-207)$ & $209(105-313)$ & $176(83-269)$ & 0.001 \\
\hline \multicolumn{6}{|l|}{ Interleukin-18(pg/ml) } \\
\hline On-admission & $301(229-373)$ & $186(151-221)$ & $200(153-246)$ & $539(46-1031)$ & 0.235 \\
\hline After $24 \mathrm{~h}$ & $220(199-240)$ & $233(151-315)$ & $200(161-238)$ & 235(184-287) & 0.891 \\
\hline After $48 \mathrm{~h}$ & 209(189-229) & $244(150-338)$ & 197(155-238) & $288(200-376)$ & 0.142 \\
\hline \multicolumn{6}{|l|}{$H s-C R P(\mathrm{ng} / \mathrm{ml})$} \\
\hline On-admission & $3.9(3.4-4.3)$ & $5.0(2.3-7.6)$ & $4.3(2.1-6.5)$ & $3.5(2.2-4.8)$ & 0.642 \\
\hline After $24 \mathrm{~h}$ & $9.6(9.1-10.1)$ & $8.6(6.1-11.1)$ & $10.6(9-12)$ & $9.6(7.7-11.5)$ & 0.601 \\
\hline After $48 \mathrm{~h}$ & $9.6(9.0-10.1)$ & $9(6-12)$ & $10.9(9.3-12.4)$ & $9.6(6.7-$ & 0.576 \\
\hline TRISS & $0.9733(0.96-0.99)$ & $0.9359(0.85-1.01)$ & $0.8207(0.67-0.97)$ & $0.9092(0.82-0.99)$ & 0.001 \\
\hline Blood transfusion & $26.1 \%$ & $18.2 \%$ & $56.3 \%$ & $31.3 \%$ & 0.062 \\
\hline Blood units transfused & $3(3-4)$ & $13(-101-127)$ & $10(1-19)$ & $18(-17-53)$ & 0.002 \\
\hline Any surgical intervention & $58 \%$ & $45 \%$ & $44 \%$ & $44 \%$ & 0.430 \\
\hline Hospital length of stay & $12(10-14)$ & $10(5-15)$ & $26(7-44)$ & $18(9-27)$ & 0.003 \\
\hline ICU length of stay & $6(4-7)$ & $10(2-17)$ & $10(6-14)$ & $8(2-14)$ & 0.106 \\
\hline Any hospital complications $(n=8)$ & $2.9 \%$ & $0 \%$ & $6.3 \%$ & $6.3 \%$ & 0.709 \\
\hline Hospital mortality $(n=12)$ & $2.9 \%$ & $18.2 \%$ & $18.8 \%$ & $6.3 \%$ & 0.005 \\
\hline
\end{tabular}

$N N$, normoglycemia non-DM: $N D$, normoglycemia DM: $S I H$, stress-induced hyperglycemia: $D H$, diabetic hyperglycemia: Continuous variables are expressed as mean and $95 \%$ confidence intervals. Comparative analysis of trauma patients based on the initial blood glucose and hemoglobin A1C was performed using one-way ANOVA tests for interval variables and chi-square tests for categorical variables

(aOR) $2.42(95 \%$ CI $1.076-5.447, p=0.03)$ for severe injury after adjusting for age, shock index and blood transfusion. However, it was not predictor for hospital mortality (aOR 0.93; 95\% CI 0.143-6.102, $p=0.94$ ) after adjusting for age, shock index, ISS, GCS and transfusion (Table 7).

\section{Discussion}

This is a prospective study to identify the patterns and effect of initial hyperglycemia and inflammatory biomarkers in trauma patients. Up to our knowledge, the constellation of on-admission random blood glucose, proinflammatory cytokines and injury severity scale among trauma patients is understudied. There are several key findings in this study. Almost $13 \%$ of trauma patients had on-admission hyperglycemia; half of them had SIH. Patients presented with hyperglycemia were more likely to have higher levels of IL-6, IL-18 and hs-CRP compared to the normoglycemic patients. Also, patients with hyperglycemia were more likely to have severe injuries, prolonged hospitalization and higher mortality than normoglycemic patients. Moreover, IL-6 levels were greater in $\mathrm{SIH}$ (non-diabetic) compared to $\mathrm{DH}$ group. Logistic regression analysis showed that adjusted on-admission blood glucose was a predictor for injury severity and not for hospital mortality.

Furthermore, this study showed that the initial readings of IL-6 correlated significantly with the patient ISS. The 

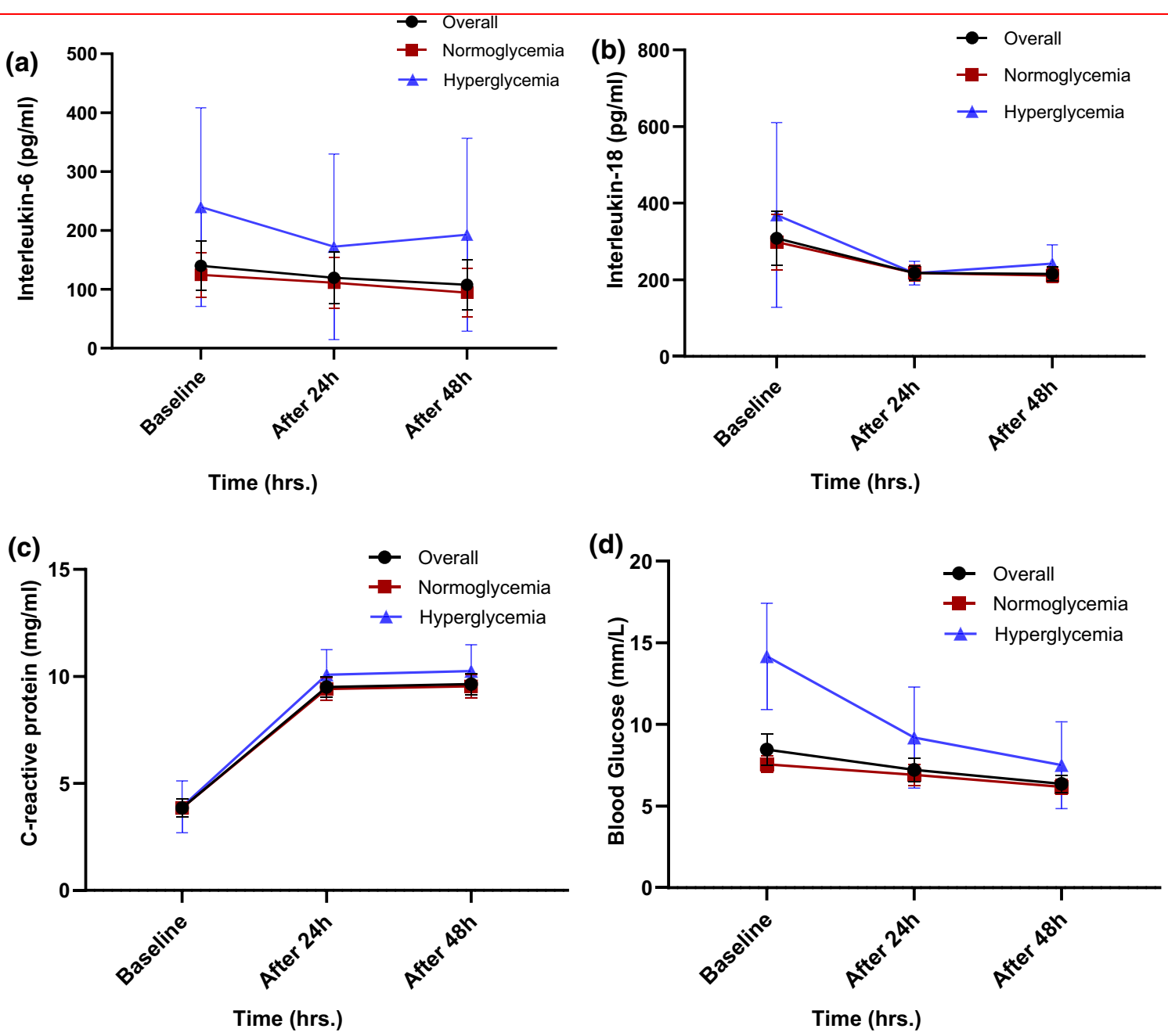

Fig. 2 Trend of inflammatory markers and blood glucose levels in the overall, normoglycemic and hyperglycemic trauma patients: a IL-6; b IL-18; c C-reactive protein; and $\mathbf{d}$ blood glucose levels

blood glucose levels showed significant correlation with serum IL-6, serum lactate, ISS and length of hospitalization. Also, we observed an association between initial hyperglycemia and hs-TnT. Prior works revealed a significant association between the severity of trauma and positivity of hs-TnT as a reflection of traumatic stress [21, 22]. Also, patients with initial hyperglycemia had a higher shock index on admission ( $>0.80$ ). Prior studies reported that trauma patients with high SI value had worse presentation and outcomes [23, 24]. Of note, the number of transfused blood units was higher in $\mathrm{DH}$ and $\mathrm{SIH}$ than normoglycemic groups in our study. Kreutziger et al., in their retrospective study, found that the rate of hemorrhagic shock was increasing with the increase in the on-admission blood glucose levels [4]. Although our study and Kreutziger et al. study had almost a similar sample size, our study was prospective study, looked for four different glycemic conditions and explored the correlation between hyperglycemia, cytokines, CRP and ISS.

The current study demonstrated that patients with SIH had a threefold higher rate of mortality as compared to those with DH. These findings are in accordance with earlier studies which showed significantly greater risk of mortality in patients with $\mathrm{SIH}$ as opposed to those who had DH $[11,15,16,25]$.

A recent study on thoracoabdominal injury patients demonstrated a higher rate of mortality in non-diabetic patients with on-admission hyperglycemia as compared to those with initial normoglycemia [26]. Furthermore, a prospective observational study of traumatic brain injury (TBI) reported a marked hyperglycemia in patients with severe TBI which independently predicted the poor shortterm neurological outcome [27]. 
Table 5 Bivariate correlation between blood glucose and other factors

\begin{tabular}{lcc}
\hline Parameters & Pearson's correlation & $p$ value \\
\hline Random glucose vs WBC at ED & 0.244 & 0.001 \\
Random glucose vs Lactate at ED & 0.467 & 0.001 \\
Random glucose vs Base Excess at ED & -0.417 & 0.001 \\
Random glucose vs GCS at ED & 0.306 & 0.001 \\
Random glucose vs ISS & 0.368 \\
Random glucose vs TRISS & -0.310 \\
Random glucose vs RTS & -0.354 \\
Random glucose vs Hospital LOS & 0.304 \\
Random glucose vs Interleukin-6* & 0.373 \\
Random glucose vs Interleukin-18* & 0.108 \\
Random glucose vs hs-CRP* & -0.059 \\
\hline
\end{tabular}

* Baseline; RTS Revised trauma score, TRISS Trauma injury severity score, ISS Injury severity score, $h s$-CRP High-sensitive C-reactive protein, $E D$ Emergency department, LOS Length of stay, Bivariate correlation coefficients were calculated between glucose and other factors

Table 6 Presentation of inflammatory markers and glucose level based on Injury Severity Score (ISS)

\begin{tabular}{|c|c|c|c|c|}
\hline Parameters & Mild $($ ISS $\leq 8) n=53$ & Moderate (ISS 9-15) $n=112$ & Severe (ISS $\geq 16) n=84$ & $p$ value \\
\hline \multicolumn{5}{|l|}{ Blood glucose level $(\mathrm{mmol} / \mathrm{l})$} \\
\hline Random (on admission) & $7.21 \pm 1.66$ & $8.05 \pm 2.69$ & $9.59 \pm 3.45$ & 0.001 \\
\hline Fasting (after $24 \mathrm{~h}$ ) & $6.65 \pm 1.41$ & $6.99 \pm 1.75$ & $7.85 \pm 3.05$ & 0.004 \\
\hline Fasting (after $48 \mathrm{~h}$ ) & $6.11 \pm 1.30$ & $6.36 \pm 1.46$ & $6.49 \pm 2.17$ & 0.48 \\
\hline HbA1c \% & $5.35 \pm 0.55$ & $5.61 \pm 1.14$ & $5.76 \pm 1.44$ & 0.14 \\
\hline \multicolumn{5}{|l|}{ Serum lactate $; \mathrm{mmol} / \mathrm{L}$} \\
\hline Baseline (on-admission) & $2.71 \pm 1.34$ & $2.71 \pm 1.11$ & $3.29 \pm 1.77$ & 0.01 \\
\hline Second day (after 24 h) & $1.82 \pm 0.95$ & $1.64 \pm 0.85$ & $2.18 \pm 1.86$ & 0.02 \\
\hline Third day (after 48 h) & $1.22 \pm 0.72$ & $1.25 \pm 0.52$ & $1.48 \pm 1.45$ & 0.24 \\
\hline \multicolumn{5}{|l|}{ Base Excess } \\
\hline Baseline (on-admission) & $-2.01 \pm 3.06$ & $-1.85 \pm 2.45$ & $-4.67 \pm 4.19$ & 0.001 \\
\hline Second day (after 24 h) & $-0.75 \pm 2.00$ & $-0.72 \pm 2.46$ & $-3.03 \pm 3.84$ & 0.001 \\
\hline Third day (after 48 h) & $1.40 \pm 2.34$ & $1.01 \pm 2.53$ & $-1.79 \pm 4.50$ & 0.001 \\
\hline \multicolumn{5}{|l|}{ Interleukin-6 (pg/ml) } \\
\hline Baseline (on-admission) & $61.4(5.8-510.3)$ & $75.9(4.8-595.9)$ & $146.6(4.02-627.1)$ & 0.001 \\
\hline Second day (after $24 \mathrm{~h}$ ) & $47.6(3.1-374.1)$ & $48.6(6.7-722.7)$ & $85.6(5.3-1109.3)$ & 0.001 \\
\hline Third day (after 48 h) & $37.3(2.4-547.9)$ & $48.6(1.9-550.0)$ & $74.5(3.2-550.0)$ & 0.04 \\
\hline \multicolumn{5}{|l|}{ Interleukin-18 (pg/ml) } \\
\hline Baseline (on-admission) & $191.6(51.9-1214.3)$ & $186.8(32.4-3890.9)$ & $197.5(45.0-5000.0)$ & 0.94 \\
\hline Second day (after 24 h) & $184.9(50.6-1328.9)$ & $183.5(41.3-739.9)$ & $211.1(47.1-737.4)$ & 0.20 \\
\hline Third day (after 48 h) & $170.3(30.3-785.6)$ & $178.5(1.04-947.9)$ & $197.7(55.0-705.1)$ & 0.07 \\
\hline \multicolumn{5}{|l|}{$h s-C R P(\mathrm{ng} / \mathrm{ml})$} \\
\hline Baseline (on-admission) & $3.05(0.06-13.1)$ & $3.09(0.18-13.5)$ & $3.35(0.03-12.8)$ & 0.55 \\
\hline Second day (after 24 h) & $10.8(0.8-14.9)$ & $10.8(0.007-14.9)$ & $10.6(0.09-13.9)$ & 0.22 \\
\hline Third day (after 48 h) & $10.9(0.02-15.5)$ & $10.9(0.004-14.3)$ & $10.7(0.2-14.9)$ & 0.56 \\
\hline All complications & $1(1.9 \%)$ & $0(0.0 \%)$ & $7(8.3 \%)$ & 0.004 \\
\hline Hospital length of stay; days & $6.0(2-62)$ & $6.5(2-68)$ & $11.5(2-146)$ & 0.001 \\
\hline In-hospital mortality & $0(0.0 \%)$ & $0(0.0 \%)$ & $12(14.3 \%)$ & 0.001 \\
\hline
\end{tabular}

One-way ANOVA for interval variables and Chi-square test 
Table 7 Multivariable logistic regression analysis for predictors of injury severity and in-hospital mortality

\begin{tabular}{llcl}
\hline Variable & $p$ value & Odds ratio & $95 \%$ confidence interval \\
\hline a-Predictors of injury severity (ISS > 12) & & & $0.981-1.032$ \\
Age; years & 0.642 & 1.006 & $0.376-1.647$ \\
Shock index & 0.523 & 0.786 & $0.882-2.765$ \\
Blood transfusion & 0.126 & 1.562 & $1.076-5.447$ \\
Admission hyperglycemia & 0.033 & 2.421 & $1.003-1.179$ \\
b-Predictors of hospital mortality & & & $0.099-4.683$ \\
Age; years & 0.041 & 1.088 & $0.143-6.102$ \\
Blood transfusion & 0.695 & 0.680 & $0.615-0.869$ \\
Admission hyperglycemia & 0.943 & 0.934 & $1.025-1.180$ \\
Glasgow Coma Scale on admission & 0.001 & 0.731 & $0.883-5.167$ \\
Injury Severity Score & 0.008 & 1.100 & 2.135 \\
Shock index & 0.092 & \\
\hline
\end{tabular}

Prior studies showed a relationship between serum cortisol, catecholamine levels and severity of injury. Patients with severe injuries were more likely to develop SIH [5, 28-30]. Consistent with these observations, hyperglycemia patients in our cohort had a higher injury severity as compared to normoglycemic patients.

In our study, the overall complications were higher in both types of hyperglycemia compared to normoglycemia groups, but the difference did not reach statistical significance. A prior study reported that initial hyperglycemia in trauma patients correlated with serum lactate and ISS and was associated with higher mortality; however, the rate of infection was not significantly higher [31]. The mortality in the present study cohort is $4.8 \%$ which is consistent with the overall in-hospital mortality among trauma patients in Qatar (4.3\%) between 2010 and 2018. [32]

In our patients, the increase in hs-CRP was detected after $24 \mathrm{~h}$ of trauma and reached its peak value at $48 \mathrm{~h}$. Giannoudis et al. [33] reported that serum CRP levels were within the normal range on the initial presentation which then gradually increased and reached the peak value on the third day post-trauma. The authors also found an association between ISS and IL-6 levels but such association was not observed with CRP. Consistent with our study, earlier studies reported a significant correlation between higher ISS and IL-6 level on the initial presentation [34-36]. We also observed a higher level of IL-18 in DH in comparison with the other groups including SIH (but statistically nonsignificant); a finding that needs further explanation [37]. Our study performed serial measurements of blood glucose and cytokines to understand the complex relationship between hyperglycemia and inflammatory response in trauma. These findings indicated that immunoneuroendocrine alterations might be involved in the pathophysiology of trauma patients [14, 15, 20].

\section{Limitations}

The first limitation is that patients with DH may have also some degree of stress response which was underestimated. Second, we could not measure the levels of stress response hormones or catecholamines. Third, selection bias cannot be ignored along the study period. The impact of hyperglycemia in female patients was not well studied as $98 \%$ of the study subjects were males. This figure reflects the gender discrepancy in trauma patients in Qatar as $90 \%$ of all trauma admissions in previous reports were males [38, 39]. Furthermore, based on the Qatar's mid-year population estimates (2014-2017), females make up almost a quarter of the total country population [40]. In the emergency department, we were using sliding scale insulin for patients with hyperglycemia; however, we did not measure the effect of exogenous insulin on the cytokine's levels. Apart from the significant difference in age, we could not explain the mortality rate in the normoglycemic diabetic group which was relatively similar to that of the SIH. Finally, the HbA1c level might not be accurate in patients who necessitate early blood transfusion as transfusion may alter the $\mathrm{HbA1c}$ reading [41, 42]. Of note, HbA1c value was reported as \% according to the Diabetes Control and Complications Trial units as we are not using the mmols/mol values (International Federation of Clinical Chemistry units) in our laboratory.

\section{Conclusion}

Patients with on-admission hyperglycemia have more severe injury and worse hospital outcome compared to normoglycemia patients. The initial blood glucose correlates with serum IL-6 which indicates a potential role of the 
systemic inflammatory response in the disease pathogenesis among severely injured patients. On-admission glucose level could be a useful marker of injury severity, triage and risk assessment in trauma patients. These observations warrant further evaluation in larger multicenter studies.

Acknowledgements We thank the national trauma registry team and trauma nurses for their cooperation. Also, we thank Prof Rajvir Singh for his effort in the statistical analysis and Mr Vinod Kumar from the Translational Research Laboratory Institute, Hamad Medical Corporation.

Author Contributions All authors have contributed substantially in the study design, data collection, data interpretation, writing draft, reviewing and approval of the manuscript.

Funding Open access funding provided by the Qatar National Library. None

Availability of Data and Material All data were shown in the study analysis and tables.

\section{Declaration}

Conflict of interests The authors declare that they have no conflict of interest.

Ethical Approval The Institutional Review Board (IRB\# 14471/14) of Hamad Medical Corporation has approved this study.

\section{Consent for Publication Not applicable}

Open Access This article is licensed under a Creative Commons Attribution 4.0 International License, which permits use, sharing, adaptation, distribution and reproduction in any medium or format, as long as you give appropriate credit to the original author(s) and the source, provide a link to the Creative Commons licence, and indicate if changes were made. The images or other third party material in this article are included in the article's Creative Commons licence, unless indicated otherwise in a credit line to the material. If material is not included in the article's Creative Commons licence and your intended use is not permitted by statutory regulation or exceeds the permitted use, you will need to obtain permission directly from the copyright holder. To view a copy of this licence, visit http://creativecommons. org/licenses/by/4.0/.

\section{References}

1. Eakins J (2009) Blood glucose control in the trauma patient. J Diabetes Sci Technol 3(6):1373-1376

2. Olariu E, Pooley N, Danel A, Miret M, Preiser JC (2018) A systematic scoping review on the consequences of stress-related hyperglycaemia. PLoS ONE 13(4):e0194952

3. Winkelmann M, Butz AL, Clausen JD et al (2019) Admission blood glucose as a predictor of shock and mortality in multiply injured patients. SICOT J 5:17. https://doi.org/10.1051/sicotj/ 2019015

4. Kreutziger J, Rafetseder A, Mathis S, Wenzel V, El Attal R, Schmid S (2015) Admission blood glucose predicted haemorrhagic shock in multiple trauma patients. Injury 46(1):15-20. https://doi.org/10.1016/j.injury.2014.09.018
5. Chang MW, Huang CY, Liu HT, Chen YC, Hsieh CH (2018) Stress-induced and diabetic hyperglycemia associated with higher mortality among intensive care unit trauma patients: cross-sectional analysis of the propensity score-matched population. Int $\mathbf{J}$ Environ Res Public Health 15(5):992. https://doi.org/10.3390/ ijerph15050992

6. Xiu F, Stanojcic M, Diao L, Jeschke MG (2014) Stress hyperglycemia, insulin treatment, and innate immune cells. Int $\mathrm{J}$ Endocrinol. https://doi.org/10.1155/2014/486403

7. van der Horst IC, Gans RO, Zijlstra F, Ligtenberg JJ (2004) Exogenous insulin and hypoglycemia as prognostic factors in critically ill patients. JAMA 291(5):558-559

8. Shi J, Dong B, Mao Y et al (2016) Review: traumatic brain injury and hyperglycemia, a potentially modifiable risk factor. Oncotarget 7(43):71052-71061. https://doi.org/10.18632/oncotarget. 11958

9. Greci LS, Kailasam M, Malkani S, Katz DL, Hulinsky I, Ahmadi R, Nawaz H (2003) Utility of HbA1c levels for diabetes case finding in hospitalized patients with hyperglycemia. Diabetes Care 26(4):1064-1068. https://doi.org/10.2337/diacare.26.4.1064

10. Kopelman TR, O'Neill PJ, Kanneganti SR, Davis KM, Drachman DA (2008) The relationship of plasma glucose and glycosylated hemoglobin A1C levels among nondiabetic trauma patients. J Trauma 64(1):30-3

11. Ray B, Ludwig A, Yearout LK, Thompson DM, Bohnstedt BN (2017) Stress-induced hyperglycemia after spontaneous subarachnoid hemorrhage and its role in predicting cerebrospinal fluid diversion. World Neurosurg 100:208-215

12. Nakamura M, Oda S, Sadahiro T, Watanabe E, Abe R, Nakada TA, Morita Y, Hirasawa H (2012) Correlation between high blood IL-6 level, hyperglycemia, and glucose control in septic patients. Crit Care 16(2):R58. https://doi.org/10.1186/cc11301

13. Yamashita H, Aoyama-Ishikawa M, Takahara M, Yamauchi C, Inoue T, Miyoshi M, Maeshige N, Usami M, Nakao A, Kotani J (2015) Endogenous interleukin 18 suppresses hyperglycemia and hyperinsulinemia during the acute phase of endotoxemia in mice. Surg Infect Larchmt 16(1):90-96. https://doi.org/10.1089/sur. 2013.269 (Epub 2015 Feb 4)

14. Kajbaf F, Mojtahedzadeh M, Abdollahi M (2007) Mechanisms underlying stress-induced hyperglycemia in critically ill patients. Therapy 4(1):97-106

15. Kerby JD, Griffin RL, MacLennan P, Rue LW 3rd (2012) Stressinduced hyperglycemia, not diabetic hyperglycemia, is associated with higher mortality in trauma. Ann Surg 256(3):446-452

16. Bosarge PL, Shoultz TH, Griffin RL, Kerby JD (2015) Stressinduced hyperglycemia is associated with higher mortality in severe traumatic brain injury. J Trauma Acute Care Surg 79(2):289-294. https://doi.org/10.1097/TA.0000000000000716

17. Griesdale DE, Tremblay MH, McEwen J, Chittock DR (2009) Glucose control and mortality in patients with severe traumatic brain injury. Neurocrit Care 11(3):311-316

18. American Diabetes Association. Diagnosis and classification of diabetes mellitus [published correction appears in Diabetes Care. 2010 Apr;33(4): e57]. Diabetes Care (2010): 33 Suppl 1(Suppl 1):S62-S69. doi:https://doi.org/10.2337/dc10-S062

19. Dungan KM, Braithwaite SS, Presier JC (2009) Stress hyperglycaemia. Lancet 373:1798-1807

20. Laird AM, Miller PR, Kilgo PD et al (2004) Relationship of early hyperglycemia to mortality in trauma patients. J Trauma 56:1058-1062

21. El-Menyar A, Asim M, Latifi R, Bangdiwala SI, Al-Thani H (2018) Predictive value of positive high-sensitivity troponin $\mathrm{T}$ in intubated traumatic brain injury patients. $\mathrm{J}$ Neurosurg 129(6):1541-1549

22. Mahmood I, El-Menyar A, Dabdoob W et al (2016) Troponin T in patients with traumatic chest injuries with and without cardiac 
involvement: insights from an observational study. N Am J Med Sci 8(1):17-24

23. El-Menyar A, Goyal P, Tilley E, Latifi R (2018) The clinical utility of shock index to predict the need for blood transfusion and outcomes in trauma. J Surg Res 227:52-59

24. El-Menyar A, Abdelrahman H, Alhammoud A et al (2019) Prognostic role of shock index in traumatic pelvic fracture: a retrospective analysis. J Surg Res 243:410-418

25. Rau CS, Wu SC, Chen YC, Chien PC, Hsieh HY, Kuo PJ, Hsieh $\mathrm{CH}$ (2017) Higher mortality in trauma patients is associated with stress-induced hyperglycemia, but not diabetic hyperglycemia: a cross-sectional analysis based on a propensity-score matching approach. Int J Environ Res Public Health. https://doi.org/10. 3390/ijerph14101161

26. Su WT, Wu SC, Chou SE, Huang CY, Hsu SY, Liu HT, Hsieh CH (2019) Higher mortality rate in moderate-to-severe thoracoabdominal injury patients with admission hyperglycemia than nondiabetic normoglycemic patients. Int $\mathrm{J}$ Environ Res Public Health 16(19):3562. https://doi.org/10.3390/ijerph16193562

27. Harun Haron R, Imran MK, Haspani MS (2011) An Observational study of blood glucose levels during admission and 24 hours post-operation in a sample of patients with traumatic injury in a hospital in Kuala Lumpur. Malays J Med Sci 18(4):69-77

28. Marik PE (2009) Critical illness-related corticosteroid insufficiency. Chest 135(1):181-193

29. Leto R, Desruelles D, Gillet JB, Sabbe MB (2015) Admission hyperglycaemia is associated with higher mortality in patients with hip fracture. Eur J Emerg Med 22:99-102

30. Sung J, Bochicchio GV, Joshi M, Bochicchio K, Tracy K, Scalea TM (2005) Admission hyperglycemia is predictive of outcome in critically ill trauma patients. J Trauma 59(1):80-83. https://doi. org/10.1097/01.ta.0000171452.96585.84

31. Duane TM, Dechert T, Dalesio N et al (2006) Is blood sugar the next lactate? Am Surg 72(7):613-618

32. El-Menyar A, Mekkodathil A, Asim M, Consunji R, Strandvik G, Peralta R, Rizoli S, Abdelrahman H, Mollazehi M, Parchani A, Al-Thani H (2020) Maturation process and international accreditation of trauma system in a rapidly developing country. PLoS One 15(12): 0243658

33. Giannoudis PV, Smith MR, Evans RT, Bellamy MC, Guillou PJ (1998) Serum CRP and IL-6 levels after trauma not predictive of septic complications in 31 patients. Acta Orthop Scand 69(2):184-8. https://doi.org/10.3109/17453679809117625

34. Gebhard F, Pfetsch H, Steinbach G et al (2000) Is IL-6 an early marker of injury severity following major trauma in humans? Arch Surg 135:291-295

35. Pape HC, van Griensven M, Rice J et al (2001) Major secondary surgery in blunt trauma patients and perioperative cytokine liberation: determination of the clinical relevance of biochemical markers. J Trauma 50:989-1000

36. Akkose S, Ozgurer A, Bulut M, Koksal O, Ozdemír F, Ozguç H (2007) Relationships between markers of inflammation, severity of injury, and clinical outcomes in hemorrhagic shock. Adv Ther 24(5):955-962. https://doi.org/10.1007/BF02877699

37. Zaharieva E, Kamenov Z, Velikova T, Tsakova A, El-Darawish Y, Okamura H (2018) Interleukin-18 serum level is elevated in type 2 diabetes and latent autoimmune diabetes. Endocr Connect 7(1):179-185. https://doi.org/10.1530/EC-17-0273

38. Arumugam S, Al-Hassani A, El-Menyar A, Abdelrahman H, Parchani A, Peralta R et al (2015) Frequency, causes and pattern of abdominal trauma: a 4-year descriptive analysis. J Emerg Trauma Shock 8(4):193-198. https://doi.org/10.4103/0974-2700. 166590

39. El-Menyar A, El-Hennawy H, Al-Thani H, Asim M, Abdelrahman H, Zarour A et al (2014) Traumatic injury among females: does gender matter? J Trauma Manag Outcomes 28(8):8. https:// doi.org/10.1186/1752-2897-8-8

40. https://www.psa.gov.qa/en/statistics/Statistical\%20Releases/ Social/GenrealSocialStatistics/MenWomenProfile/2018/Woman Man_2018_EN.pdf. Accessed on 29 May 2021

41. Spencer DH, Grossman BJ, Scott MG (2011) Red cell transfusion decreases hemoglobin A1c in patients with diabetes. Clin Chem 57(2):344-346

42. Farrokhi F, Smiley D, Umpierrez GE (2011) Glycemic control in non-diabetic critically ill patients. Best Pract Res Clin Endocrinol Metab 25(5):813-824

Publisher's Note Springer Nature remains neutral with regard to jurisdictional claims in published maps and institutional affiliations. 